$\mathrm{H}_{19}$. Brennstoffe. Erdöl. Mineralöle

A. N. Glaskow und A. N. Parfenow, Die elektrischen Anlagen der Erdöl- und Gasverarbeitungswerke. Moskau: Gosstoptechisdat. 1962. (343 S. m. Abb.) 90 Kop. [russ.]

Werner Hansen, 1000 Fachwörter für die Olfeuertechnik. Englisch-deutsch. Deutsch-englisch. Stuttgart: Gentner. 1962. (66 S.) $8^{\circ}=$ AW G-Reihe, Bd. 122. Feuerungstechnik. DM 7,80.

Eduard Kersten u. a., Kolloquium Heizole vom 8. und 9. Dezember 1960 in Markkleeberg. Brennstofftechnische Gesellschaft in der Deutschen Demokratischen Republik. Berlin: Akademie-Verl. 1962. $\left(166 \mathrm{~S} . \mathrm{m} .80 \mathrm{Abb}\right.$. u. 33 Tab.) $8^{\circ}=$ Freiberger Forschungshefte. Reihe A. 215. DM 20,-

Nilio Oksala, Asfalttipăăllysteiden stabililisuuteen vaikuttavista tekijőistä. (Uber die Faktoren mit EinfluB auf die Stabilităt von Asphaltbelägen). Helsinki: Valtion Teknillinen Tutkimuslaitos. 1962. (61 s. m. Abb.) $8^{\circ}$.

W. A. Oleinlkow und N. Ss. Sotow, Die automatische Regulierung technologischer Prozesse in der Erdölund petrolchemischen Industrie. Leningrad: Gosstoptechisdat, Leningrader Abt. 1962. (323 S. m. Zeichng.) 1 Rbl. 9 Kop. [russ.]

G. P. Owanessow, Die Blldung von Erdöl- und Gaslagerstätten in Baschkirien, ihre Klassifikation und Schürfmethoden. Moskau: Gosstoptechisdat. 1962. (295 S. m. Zeichng. u. Kart.; 4 Bl. Kart.) 1 Rbl. 60 Kop. [russ.]

Ja. M. Wassiljew und O. Ss. Obrjadtschlkow, Perspektiven der Gas-Erdölfründigkeit von Pliozän-Ablage rungen in der Kaspischen Mulde. Moskau: Gosstoptechisdat. 1962.' (180 S. m. Abb. u. Kart.) 1 Rbl. 4 Kop. [russ.]

$\mathbf{H}_{21}$. Leder. Gerbstoffe

-, Determination of the colour fastness of leather. London: British Standards Institution. 1962. (52 S.) $15 \mathrm{s.}$

\title{
Ausgewertete Bibliographie-Fachzeitschriften
}

Belgien

Bibliographie de Belgique - Belgische Bibllographio

CssR

Bibliography of Publications Dealing with the Polarography Method [Praha]

Bulletin - Die Neuesten Forschungsergebnisse der Tschechoslowakischen Wissenschaft

Nové Knihy (Neue Bücher)

Novinky (Neuheiten)

Dănemark Danske Bogmarked

Deutschland Borrsenblatt für den Deutschen Buchhandel - Frankfurter Ausgabe

Börsenblatt für den Deutschen Buch. handel - Leipziger Ausgabe

Deutsche Bibliographie. Wöchentliches Verzeichnis. Ausgabe II. [Frankfurt/Main]
Deutsche Nationalbibliographie. Reihe A: Neuerscheinungen der Buchhandels [Leipzig]

England British National Bibliography

Frankreich Bibliographie de la France

Griechenland Greek Bibliography [Athen]

Italien Bibliografia Nazionale Italiana

Niederlande Nieuwsblad voor de Boekhandel

Osterreich Osterreichische Bibliographie

Schweiz Schweizer Buch. Serie A und Serie B

UdSSR Bücherverzeichnis des Jahres

(Книжная летопись

Neue Bücher (Новые книги)

USA Publishers' Weekly 